\title{
Flocculation of microfibrillated cellulose in shear flow
}

\author{
Anni Karppinen - Tapio Saarinen • \\ Juha Salmela • Antti Laukkanen • \\ Markus Nuopponen · Jukka Seppälä
}

Received: 23 March 2012/ Accepted: 9 August 2012/Published online: 18 August 2012

(C) Springer Science+Business Media B.V. 2012

\begin{abstract}
In this work, the rheological properties of microfibrillated cellulose suspensions under stepped flow and constant shear were studied using a combination of rotational dynamic rheometer and digital imaging. During each rheological measurement, the structure of the suspension was monitored through a transparent outer cylinder with a digital camera. This enabled simultaneous analysis of the suspension floc size distribution and traditional rheological characterization. In stepped flow conditions, a good correlation between suspension floc structure and flow curve measurement was found. At constant shear, the suspension structure was dependent on the shear rate and concentration of the suspension. A low shear rate resulted in heterogeneous floc structure, which was also detected by an increase in the ratio of the viscous component to elastic component in the rheological measurement. At low concentrations and $0.5 \mathrm{~s}^{-1}$ shear rate, flow induced a formation of floc
\end{abstract}

A. Karppinen · T. Saarinen · J. Seppälä $(\square)$

Polymer Technology, Department of Biotechnology

and Chemical Technology, Aalto University,

P.O. Box 16100, 00076 Aalto, Finland

e-mail: jukka.seppala@aalto.fi

J. Salmela

VTT, Koivurannantie 1, P.O. Box 1603,

40101 Jyväskylä, Finland

A. Laukkanen · M. Nuopponen

UPM, New Businesses and Development, Tekniikantie

2C, 02150 Espoo, Finland cylinders between the rotating cylinder and stationary cup surface.

Keywords Microfibrillated cellulose - Rheology · Floc structure $\cdot$ Image analysis $\cdot$ Flow curve

\section{Introduction}

Microfibrillated cellulose (MFC) is a unique nanomaterial that is prepared by the disintegration of wood or other cellulosic fibers into fibrils. It was first introduced by Turbak et al. (1983) and Herrick et al. (1983) in the 1980 s and, at that time, prepared purely by mechanical disintegration. Today, oxidation (Saito et al. 2006) or enzymatic (Pääkkö et al. 2007) pretreatments can be used prior to mechanical treatment to lower the energy demand in the disintegration step and to modify the fiber surface. Depending on the manufacturing procedure, the fibers may be single fibrils but most commonly they consist of fibrils and fibril aggregates having a diameter between 5 and $30 \mathrm{~nm}$ and a high aspect ratio (Vartiainen et al. 2011).

MFC fibers form an entangled, three-dimensional network (Pääkkö et al. 2007) and the structure is flocculated in water suspensions. The floc structure is to some extent similar to pulp suspensions (Saarikoski et al. 2012); although MFC fibers have significantly smaller dimensions and a higher aspect ratio than pulp 
fibers. The flocculated structure of MFC (Saarikoski et al. 2012) and pulp suspensions (Beghello 1998; Wågberg and Nordqvist 1999; Chen et al. 2002; Björkman 2003a, b) have been studied by imaging. At rest, the flocs can be described as areas of higher fiber density within a network (Björkman 2000; Kerekes 2006). Under shear, the network breaks gradually, and at sufficiently high shear rates, the suspension flows as individual flocs (Björkman 2006; Saarikoski et al. 2012), and the floc size diminishes with increasing shear rate (Björkman 2005; Saarikoski et al. 2012). The floc strength is determined by number of contacts and cohesive forces present between the fibers (Kerekes et al. 1985). The cohesive forces are in this case mechanical or colloidal. The fibers can collide and entangle mechanically to form flocs under flow. On the other hand, flow may break down the flocs by eroding fibers from the surface or by splitting the floc in two (Björkman 2000; Hubbe 2007).

Colloidal factors contributing to flocculation can be described by DLVO theory, first introduced by Derjaguin and Landau (1941) and Verwey and Overbeek (1948). These forces consist of van der Waals interactions and electrostatic double layer interactions caused by surface charges. The van der Waals forces are attractive between similar surfaces, whereas the electrostatic double layer interactions are repulsive between like-charged surfaces (Hubbe and Rojas 2008). Since MFC has slightly negatively charged surface in aqueous environment (Pääkkö et al. 2007; Eronen et al. 2011), electrostatic double layer causes repulsion between fibers at longer distance if the electrolyte concentration is low, whereas attractive van der Waals forces are important at closer distance. In addition to these, flocculation can be affected by non-DLVO interactions like steric and electrosteric interactions caused by polymers and polyelectrolytes in the system (Hubbe and Rojas 2008). All these forces and thus the floc structure can be changed for example by salt addition (Ono et al. 2004; Saarikoski et al. 2012), polyelectrolyte addition (Wågberg and Nordqvist 1999; Hubbe 2007; Karppinen et al. 2011) or by changing the pH (Beghello 1998). The suspension structure and the related rheological properties are important for the processing of MFC, e.g., pumping or storage, and in some applications, e.g., use as a stabilizer in dispersions or rheology modifier in paints and coatings.

The strong interfibrillar forces in the MFC suspension result in gel-like behavior and other interesting rheological properties even at low concentrations $(0.125 \%)$ (Pääkkö et al. 2007). MFC has high viscosity at low shear rates, and it shows significant shear thinning with increasing shear rates (Pääkkö et al. 2007; Agoda-Tandjawa et al. 2010; Iotti et al. 2011). In addition to this, some researchers have observed two shear thinning regions with a viscosity plateau between them (Agoda-Tandjawa et al. 2010; Iotti et al. 2011; Karppinen et al. 2011) and a shear rate-viscosity hysteresis loop when measuring a flow curve as a function of shear rate (Iotti et al. 2011). These characteristics have been suggested to be due to structural changes in the MFC suspension microstructure (Agoda-Tandjawa et al. 2010; Iotti et al. 2011; Karppinen et al. 2011), although no unambiguous microstructure for the suspension is known.

In general, microstructures can be formed by flocculated particles, alignment of fibers, favorable spatial distributions of particles or drops, and entanglement or association density in polymer solutions (Barnes 1997). Microstructures determine the macroscopic rheological properties of the suspensions, like viscosity and elasticity. In the colloidal suspension, Brownian motion, attractive and repulsive forces (dispersion, electrostatic, and steric forces) affect the microstructure in the absence of flow (Mewis and Wagner 2009). Other important properties in the case of rigid rods are the aspect ratio, particle number density, and flexibility (Solomon and Spicer 2010). On the other hand, the structure is dependent on the flow, and the adaptation to changing flow conditions may take from seconds to hours. Dependence on the shear rate gives rise to shear thinning and yield stress behavior, and the time scale of accommodating the structure to the changing shear conditions causes thixotropy. Anisotropic organization of the suspension in the flow conditions may occur if the interactions between the particles are comparable to hydrodynamic forces (Vermant and Solomon 2005).

We have previously reported a new experimental design for observing suspension structure while measuring rheological properties (Saarikoski et al. 2012). The set up consists of a transparent outer geometry for the rheometer and a digital camera that is controlled by a computer. We have used it to verify the flocculated nature of MFC and study the effect of different geometry dimensions, the degree of the fiber disintegration and salt (Saarikoski et al. 2012) or polyelectrolyte addition to MFC suspension (Karppinen et al. 2011). In this paper, we concentrate on the flow curve 
characteristics of MFC suspensions, which have some interesting and not fully understood characteristics described above. We use the photographing system combined with floc size analysis of the images to explain the shape of the flow curve at different MFC concentrations. In addition, we observe the structures after constant shear and compare the structure to loss tangent (the ratio of viscous and elastic component) of the suspension.

\section{Experimental}

\section{Materials}

Microfibrillated cellulose (UPM Fibril Cellulose) was obtained from UPM-Kymmene Corporation. The material was prepared from never-dried bleached kraft birch pulp by mechanical disintegration. The pulp was changed to its sodium form and washed with deionized water to electrical conductivity less than $10 \mu \mathrm{S} / \mathrm{cm}$ according to a procedure introduced by Swerin et al. (1990) and subsequently ground five times in Supermasscolloider (Masuko Sangyo, Japan). MFC suspension was received in its sodium form, washed and ground at $2 \%(\mathrm{w} / \mathrm{w})$ concentration. Previously, the pulp used as starting material has been determined to have the negative charge density of $65 \mu \mathrm{eq} / \mathrm{g}$ by conductometric titration (Eronen et al. 2011). The resulting MFC has been reported to contain approximately $27 \%$ hemicelluloses (Eronen et al. 2011) and the fiber diameters have been measured to be between 10 and $30 \mathrm{~nm}$ with some larger fibril aggregates (Vartiainen et al. 2011). Dilution of the suspension was made with deionized Milli-Q water (electrical conductivity $<0.2 \mu \mathrm{S} / \mathrm{cm}$ at $25{ }^{\circ} \mathrm{C}$ ) in order to avoid excess electrolytes. The dilution was performed with propeller mixer (diameter $58 \mathrm{~mm}$, RZR 2051 control, Heidolph Instruments, Germany) at the speed of $1,400 \mathrm{rpm}$ for $10 \mathrm{~min}$. $\mathrm{pH}$ of the $2 \%$ suspension was 6.0 , and it increased slightly with water addition so that for $0.1 \%$ suspension $\mathrm{pH}$ was 6.4 .

\section{Rheological measurements}

The measurements were performed using a dynamic rotational rheometer (ARG2, TA Instruments), with a standard (ISO 3219/DIN 53019) metal concentric cylinders geometry (bob and cup radii 14 and $15 \mathrm{~mm}$ ).
After a $20 \mathrm{~min}$ preshear interval at $500 \mathrm{~s}^{-1}$ and a subsequent $30 \mathrm{~min}$ recovery period (time sweep at $0.5 \%$ strain and $1 \mathrm{~Hz}$ frequency), frequency sweep was measured from 0.02 to $200 \mathrm{rad} / \mathrm{s}$ at $0.5 \%$ strain and amplitude sweep from the strain 0.01 to $1000 \%$ at the frequency of $6.283 \mathrm{rad} / \mathrm{s}$. The flow curve was measured strain-controlled from 0.01 to $1,000 \mathrm{~s}^{-1}$ with a point time of $15 \mathrm{~s}$. Before and after the flow curve, a time sweep at $0.5 \%$ strain and $1 \mathrm{~Hz}$ frequency was conducted for $10 \mathrm{~min}$. In addition, the suspensions were measured at four constant shear rates $\left(500,30,5\right.$, and $\left.0.5 \mathrm{~s}^{-1}\right)$ for $10 \mathrm{~min}$. After each shear rate, a 10 min oscillatory time sweep was measured at $0.5 \%$ strain and $1 \mathrm{~Hz}$ frequency. The measurements were performed at $25{ }^{\circ} \mathrm{C}$.

In addition to standard metal outer geometry, a tailormade transparent polymethyl methacrylate (PMMA) outer geometry was used in order to photograph the changes in the suspension structure during the measurements. The cup was placed into a transparent water container to prevent reflections. The temperature in the water container was not controlled, but it was assumed to be close to the room temperature. The photographs were taken with Nikon D90 camera (Nikon Corporation, Japan) with a macro objective controlled by NKRemote software (Breeze Systems Limited, UK).

Image analysis

Floc size analysis was done using the method presented by Karema et al. (2001). Uneven illumination in each image was corrected using the average image of all acquired images and all the remaining intensity variations in the corrected images are due to changes in suspension properties. The dominant factor causing these variations is the suspension's local concentration. In some individual cases, reflections from uneven illumination caused difficulties in the image analysis. For these few cases, floc size was not calculated. Otherwise, the floc size analysis was performed as described earlier by Saarikoski et al. (2012).

\section{Results and discussion}

Flow curves

Figure 1 shows the flow curves for MFC suspensions at several concentrations. Suspensions were extremely 


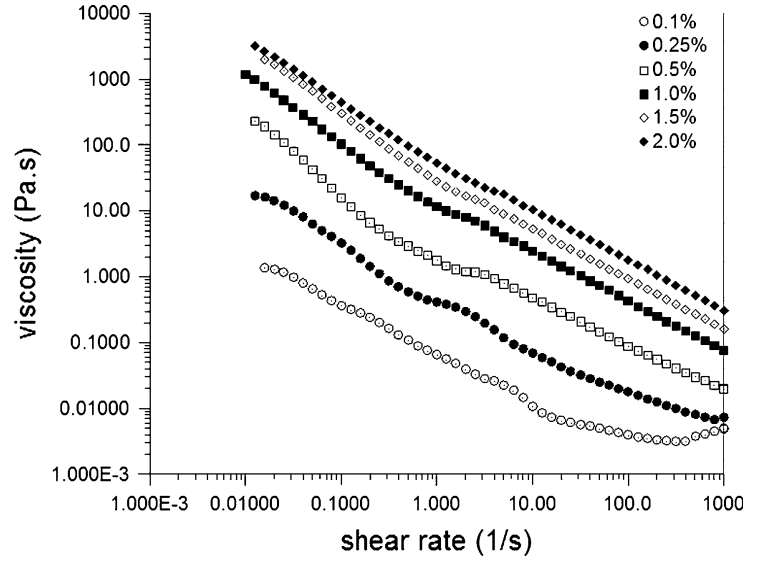

Fig. 1 Flow curves for MFC at different concentrations

shear thinning, and between the two shear thinning regions there was a viscosity plateau which has been reported for some MFC suspensions in the literature (Lowys et al. 2001; Pääkkö et al. 2007; AgodaTandjawa et al. 2010). The two shear thinning regions can be distinguished by the slope of the shear stress as a function of shear rate (Fig. 2). At low shear rates, the shear stress was constant and the viscosity was decreasing. At high shear rates, shear stress increased as a function of shear rate and a Newtonian plateau was not observed in the high shear rate region. Viscosity of the suspensions was strongly dependent on the concentration (Fig. 1), and the different regions of flow identified above were present in every concentration, although they were more pronounced for the lower concentrations. The concentration of $0.1 \%$ was close to the lowest gel forming concentration, which caused the slightly irregular shape of the flow curve, i.e., the shear stress was also increasing at low shear rates. Additionally, this concentration even showed increasing viscosity at high shear rates which could be caused by turbulent eddies.

The plateau in the flow curve at intermediate shear rates has been reported earlier for MFC (AgodaTandjawa et al. 2010; Iotti et al. 2011; Karppinen et al. 2011), and it has been observed with different measuring geometries, like plate-and-plate (AgodaTandjawa et al. 2010; Iotti et al. 2011) and concentric cylinders (Karppinen et al. 2011). However, the reason for the transition at intermediate shear rates has not been thoroughly investigated although it has been proposed to be due to structural changes (AgodaTandjawa et al. 2010; Iotti et al. 2011). In this paper,

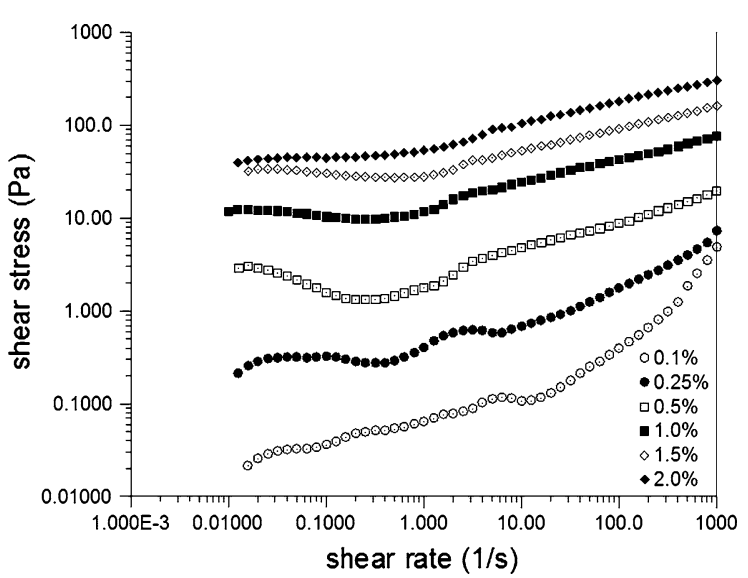

Fig. 2 Shear stress as a function of shear rate from the stepped flow measurements for several MFC concentrations

we will use imaging and image analysis to explain the flow curve characteristics of MFC suspension.

Qualitative observations of the suspension structure

The suspensions were photographed during the stepped flow measurements to study the structures related to different regions in the flow curve. Figure $3 \mathrm{a}-\mathrm{c}$ offer a qualitative view of the $1 \%$ MFC suspension floc structure at different shear rate regions. The images were chosen from the two shear thinning regions in the flow curve and from the viscosity plateau between them. At the lowest shear rates (Fig. 3a), the suspension was still visibly intact from the previous shear interval, and the flocs were small in the images. At intermediate shear rates where a plateau in viscosity was observed, the flocculated fiber network broke and flocculated structures with large flocs and voids appeared, see Fig. 3b. The voids appeared parallel to the direction of greatest compression while flocs, at first, remained chained to each other between the voids. After the breakage (Fig. 3c), the suspension remained in a flocculated state with separate, small flocs.

A closer look at the axial spiral pattern of the fiber free voids in Fig. $3 \mathrm{~b}$ reveals that the direction of the voids was approximately $45^{\circ}$ upwards or downwards from the bob rotational direction. Similar patterns have been observed in wood fiber suspensions (Björkman 2003a) and in MFC suspensions (Saarikoski et al. 2012). The explanation behind the spiral pattern has 

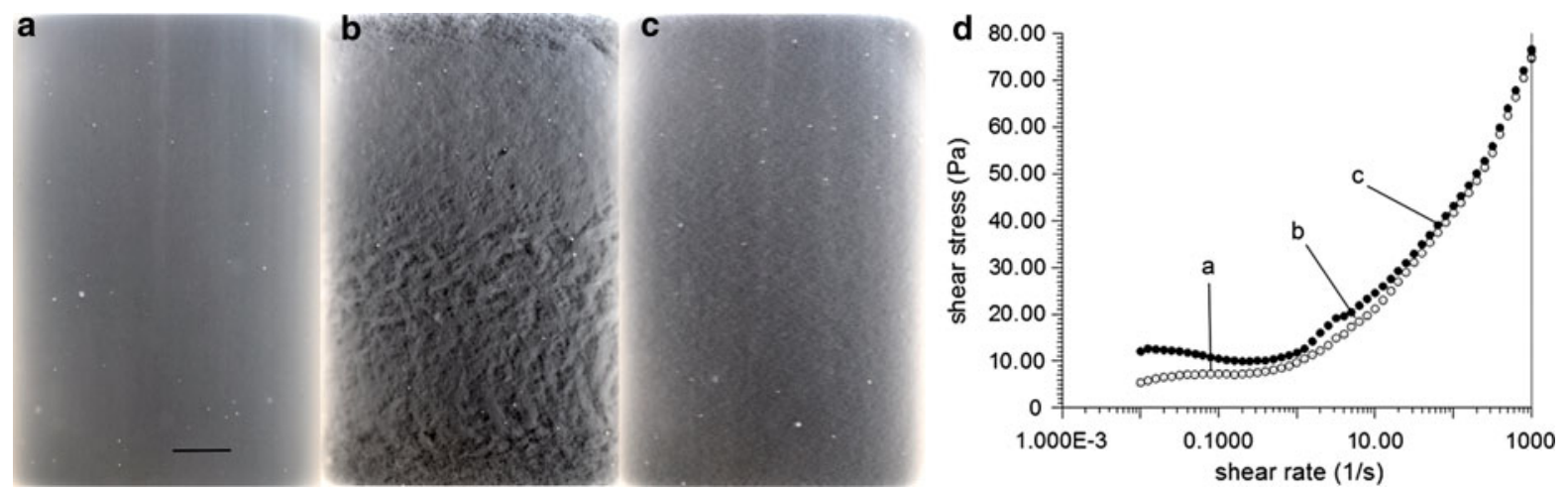

Fig. 3 Images from the flow curves for MFC suspensions at a concentration of $1 \%$. The images are from three different regions from the flow curve a shear rate $0.1 \mathrm{~s}^{-1}$ constant stress, b shear rate $5 \mathrm{~s}^{-1}$ transition region, and c shear rate $63.1 \mathrm{~s}^{-1}$

been given previously for pulp suspensions (Björkman 2003a). The start of the flow in the gap was not homogeneous, which is clearly seen in Fig. 3b. When part of the suspension begins to flow but it is still attached to stationary suspension below or above it, there is axial torsion, influencing the flowing suspension. If the flowing suspension is attached to the stationary suspension below, the flocs are extended in $45^{\circ}$ upwards from the bob rotational direction and, hence, compressed in the perpendicular direction. The compression forces out water from the flocs, inducing voids to the network. As the shear rate was increased, the flow in the gap became vertically homogeneous and there was no axial spiral pattern (Fig. 3c).

The rheograph in Fig. 3d shows the rheological results measured with the standard metal geometry and with the transparent cup and metal bob. Curves are fairly overlapped but there is a slight difference, especially at the low shear rates, probably due to the poorer stress transfer in the transparent cup. Therefore, we have always measured the samples with both the metal and transparent geometries and the results reported here are from the metal geometry measurements if it is not otherwise stated.

Floc size analysis of the flow curves

For more precise analysis of the structure's relation to the flow curve details, floc sizes were determined from the images, and the results are summarized in Fig. 4. In addition, Fig. 5 shows the representative floc size distribution at six shear rates for the $1 \%$ MFC suspension. In the images, $\mathrm{x}$ is along the direction of increasing shear stress. The inner cylinder rotates to the left. Rheograph (d) shows flow curves with the metal cup (filled circles) and the transparent cup (empty circles) and the positions of the images. The length of the scale bar is $5 \mathrm{~mm}$

flow and y perpendicular to the direction of flow. At low shear rates, floc size was independent of the shear rate and the floc size distribution was very narrow, since the network was uniform, which reflects the previous shear interval. The flocs were seen as more concentrated areas within the network, and the contacts were mostly physical entanglements and entanglements by partially disintegrated fibrils (Pääkkö et al. 2007). We assume that the shear rate was still low enough that the flocculated fiber network was able to recover from the shear and formed new points of contact between the flocs as the suspension was sheared. Therefore, the overall three-dimensional network structure was preserved and no macroscopic changes in the structure were observed.

The viscosity plateau at the intermediate shear rates and transition from the constant shear stress to the increasing stress region was related to a significant increase in floc size (Fig. 4) and broadening of the size distribution (Fig. 5), signaling heterogeneous floc structure. Such a large change in the macroscopic structure explains the viscosity plateau in the flow curve. It is evident from the images that the fiber free voids appeared in the three-dimensional floc network in this shear rate region. The voids appeared in the direction of greatest compression, first between the flocs where there are less contact points between the fibers (Kerekes et al. 1985). Shear also induced collisions and thus new contact points between the fibers in the fiber rich areas. New contacts by mechanical links between fibers are favored by two characteristics of MFC. First, MFC fibers have a high aspect ratio (Vartiainen et al. 2011), which enables 

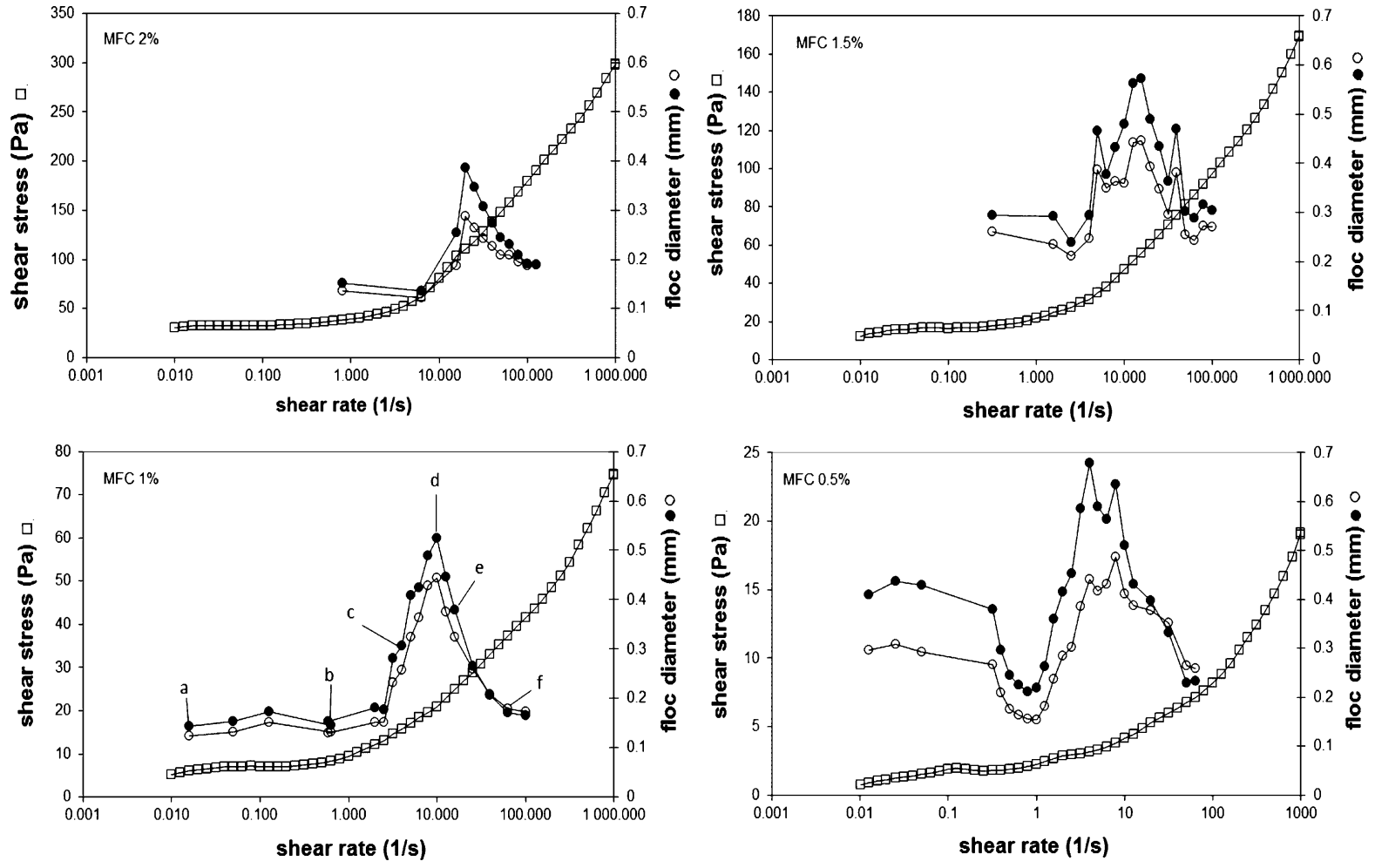

Fig. 4 Shear stress (empty squares) and floc size in $\mathrm{x}$ (empty circles) and $\mathrm{y}$ (filled circles) directions as a function of shear rate for MFC at different concentrations. Shear stress was measured with transparent geometry. The letters $\mathbf{a}-\mathbf{f}$ refer to size distributions in Fig. 5

them to bend elastically. As elastically bent fibers come to rest, they may become restrained by adjacent fibers inducing normal forces at fiber contact points and together with frictional forces inhibit movement between fibers (Kerekes et al. 1985; Kerekes 2006). Secondly, the morphology of fibers is somewhat irregular since there are also only partially fibrillated fibers (Pääkkö et al. 2007), which favors mechanical entanglements.

The negative surface charge $(65 \mu \mathrm{eq} / \mathrm{g})$ on the MFC fibers, which was mainly due to hemicelluloses, caused repulsion between the fibers as they collided with each other and prevented van der Waals interactions which would lead to permanent aggregation. The surface charge was low, but since excess ions were removed by washing, the Debye length of the electrostatic double layer is expected to be sufficient to reduce flocculation and permanent aggregation of the fibers. Earlier, it has been found that increased ionic strength causes severe aggregation in MFC or pulp suspensions (Ono et al. 2004; Saarikoski et al. 2012). Therefore, it is likely that there would have been more attractive forces between the fibers at higher ionic strengths, and thus, the plateau in the flow curve would have been more pronounced.

In the second shear thinning region, the network structure was replaced with individual flocs, and the floc size diminished with increasing shear rate (Fig. 4). The size distribution narrowed (Fig. 5), showing that the structure became rather uniform once more. This was due to the higher shear rate that started to disrupt the large flocs and broke floc chains which indicates that the contacts between the fibers were reversible. Increasing shear rate also increased the collision frequency between the fibers and flocs, forming new contacts. The balance between new contact points and disruption of the flocs determined the floc size at each shear rate. In this region, the flocs were rolling and moving relatively to each other, which enabled them to orientate themselves in the direction of flow. The decreasing floc size caused highly shear thinning behavior at the high shear rates (Barnes 1997). The narrow size distribution indicates that the floc size adapted rapidly to the changing flow environment at 
Fig. 5 Floc size distribution in $\mathrm{x}$ (empty circles) and y (filled circles) directions for $1 \%$ MFC in flow curve measurement at different shear rates: a $0.016 \mathrm{~s}^{-1}$, b $0.63 \mathrm{~s}^{-1}$, c $4.0 \mathrm{~s}^{-1}, \mathbf{d ~} 10 \mathrm{~s}^{-1}$, e $161 / \mathrm{s}$, and $\mathbf{f} 63 \mathrm{~s}^{-1}$
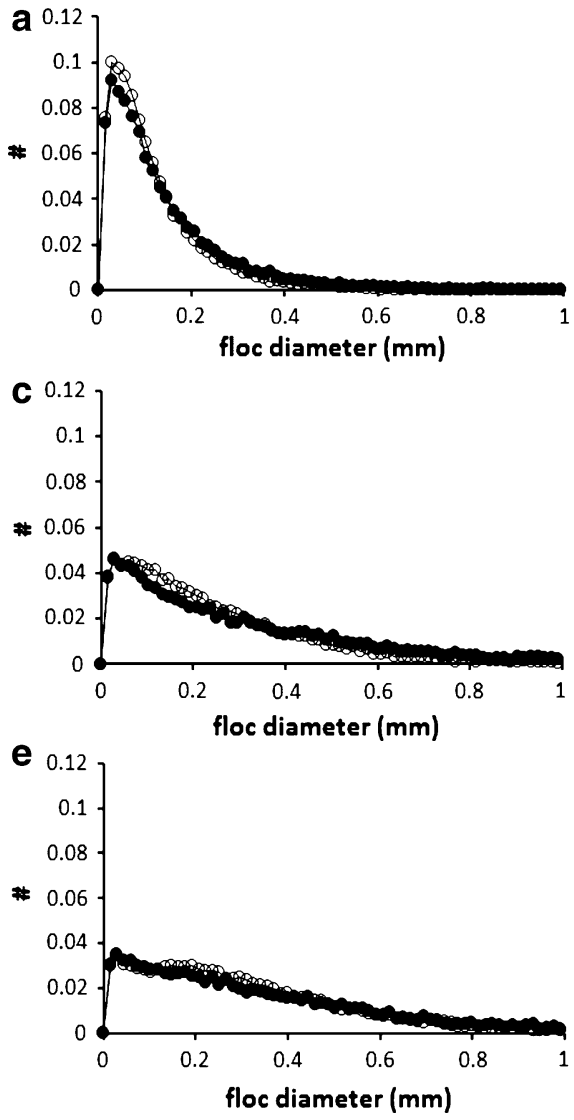
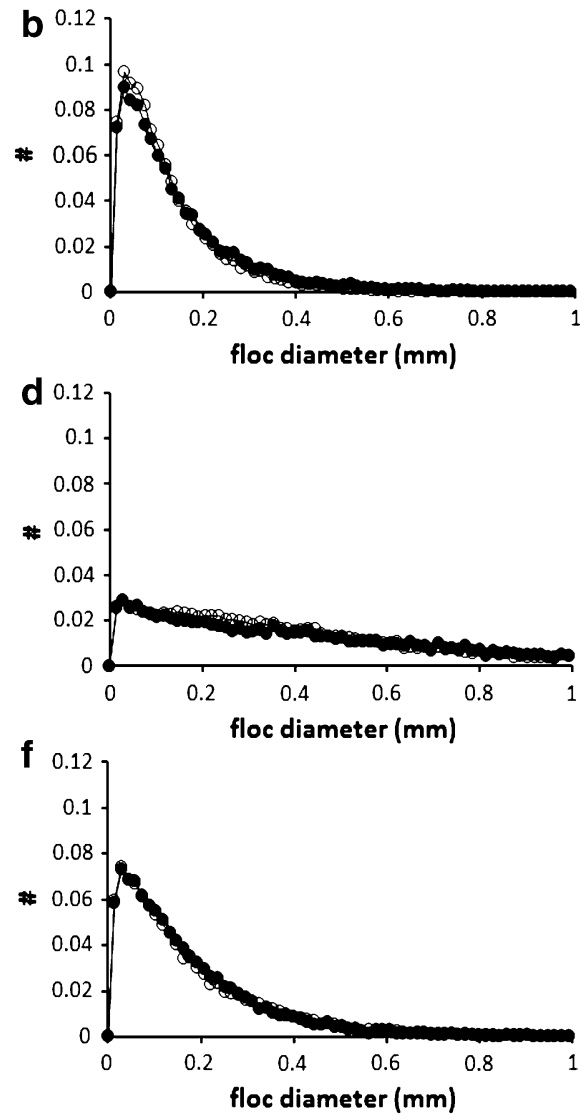

high shear rates. The floc diameter was slightly larger in the $\mathrm{y}$ direction than in the $\mathrm{x}$ direction in all suspensions and at most shear rates, aside from the highest shear rates in Fig. 4. This is explained by the rotating inner cylinder that compressed the flocs in the $\mathrm{x}$ direction and caused the elongation in the y direction.

Similar structural changes and flow curve shape have been observed earlier for attractive emulsions (Montesi et al. 2004). The flow curve of the emulsion contained two shear thinning regions and a constant viscosity region between them, similar to our suspensions. The rheological behavior of the attractive emulsions has been correlated to the structural changes observed during the measurements. In the first shear thinning regime, a roughly uniform arrangement of droplets was reported. At the transition between the shear thinning regimes, voids filled with continuous phase was observed between the flocs, followed by the appearance of cylindrical flocs. At high shear rates, the microstructure of the attractive emulsion was nearly uniform again (Montesi et al. 2004). This is in agreement with our findings and suggests that this kind of changes in the floc structure is generally related to the three regions in the flow curve.

Some restrictions were encountered in the analysis system. First, the imaging was restricted to shear rates below $100 \mathrm{~s}^{-1}$ due to the exposure time required by the camera under the given lighting conditions. Second, it seems that the floc size started to level-off in the high shear rate end, but since the viscosity did not reach a plateau within the measured shear rate range, we assume that flocs did not reach their lowest possible size in the observed region. A Newtonian region in viscosity at the high shear rate end would have indicated that the floc size did not decrease further, but it was not detected at the measured shear rates. Therefore, we assume that the floc size continued diminishing with increasing shear rate, but it occurred below the resolution of the imaging setup. Third, the image analysis gave reliable results at concentrations $0.5-2 \%$ but below $0.5 \%$ the contrast between the suspension and inner cylinder was too poor for a proper analysis of the floc size. A qualitative evaluation revealed, however, that similar structural changes 
occurred at low concentration ( 0.1 and $0.25 \%$ ) during the flow curve, as was detected at the higher concentrations. Additionally, at intermediate shear rates, flocs formed some separate floc cylinders at concentrations 0.1 and $0.25 \%$, which is discussed in more detail later in this paper. Last, the inner cylinder is moving, in relation to the camera, which means that the area that is photographed changes. This caused some irregularities in the floc size graph in the area where the suspension structures were not uniform, especially at concentrations 1.5 and $0.5 \%$ in Fig. 4.

\section{Floc size in constant shear}

The adjustment of the suspension structure to the flow conditions is time-dependent, and therefore, we studied the suspension under $10 \mathrm{~min}$ constant shear at four different shear rates. The 10 min constant shear rate was followed by a 10 min oscillatory time sweep at a very low strain $(0.5 \%)$ which is well within the linear viscoelastic region. The images were taken after stopping the shear, since otherwise the highest shear rate $\left(500 \mathrm{~s}^{-1}\right)$ could not have been photographed. When the flow was suddenly arrested, the structure froze at the macroscopic level and the observed floc structure remained constant. However, following the storage and loss moduli at rest with a time sweep measurement after the shear interval, they were observed to increase over a long period of time-signaling the optimization of fiber contact points of both inter and intra flocs (Saarikoski et al. 2012). The recovery was probably caused by bent fibers straightening after shear stops, repulsion between the slightly negative fibers driving them apart, and Brownian motion. The decreased mobility of the fibers in the flocculated network during this rest interval was also reflected in decreasing loss tangent (tan delta) values. Tan delta is the ratio of loss modulus to storage modulus. The decrease in tan delta values was greatest in the beginning and the tan delta value did not reach a constant level within $10 \mathrm{~min}$. For example, after shearing at $0.5 \mathrm{~s}^{-1}, 1 \%$ MFC suspension had a tan delta value of $0.266 \mathrm{~s}$ after the stop and tan delta value of $0.18100 \mathrm{~s}$ after the stop, and it further decreased to $0.16600 \mathrm{~s}$ after the stop.

Figure 6 offers a qualitative view of the $1 \%$ MFC suspension structure, and Fig. 7 shows the average floc sizes in the $\mathrm{x}$ and $\mathrm{y}$ directions and tan deltas at different concentrations, measured in an oscillatory time sweep after each constant shear rate. In addition, a representative size distribution is given for $1 \% \mathrm{MFC}$ suspensions in Fig. 8. The peak holds and oscillatory time sweeps were performed one after another starting from the highest shear rate, and thus the structure in the beginning of the peak hold was determined by the previous shear interval. At these four shear rates, the floc size was proportional to the preceding shear rate, except at the highest concentration of $2 \%$, where the lowest shear rate $0.5 \mathrm{~s}^{-1}$ resulted in a slightly lower average floc size than the shear rate $5 \mathrm{~s}^{-1}$. Moreover, at the concentration of $0.5 \%$, the y size was abnormally high at shear rates 30 and $500 \mathrm{~s}^{-1}$. By visual inspection, the flocs were not significantly larger in y direction, but there were vertical reflections on the cylinder which caused the high y size in the image analysis. These reflections were too dominant and impeded the image analysis, leading to a too large $y$ size in the high shear rate photos. At lower shear rates, the suspension structure was more heterogeneous and the reflections did not influence the analysis. At higher concentrations, the inner cylinder was not visible through the suspension and, therefore, reflections did not appear in the images. The floc size dependence on the shear rate has previously been reported for pulp (Björkman 2005) and MFC suspension (Saarikoski et al. 2012).

The highest shear rate $\left(500 \mathrm{~s}^{-1}\right)$ created a uniform floc structure, where the individual flocs were hard to distinguish from each other. The structure after shearing at $30 \mathrm{~s}^{-1}$ was still relatively uniform but the individual flocs were easier to observe, and the structure was coarser than after $500 \mathrm{~s}^{-1}$ shearing. This is also evident in the size distribution in Fig. 8, where the distribution broadened after shearing at $30 \mathrm{~s}^{-1}$ compared to the highest shear rate. Shear rates 5 and $0.5 \mathrm{~s}^{-1}$ were where flocs chained in a tangential spiral pattern, and the structure was heterogeneous with large and irregular floc clusters. The suspension after shearing at $0.5 \mathrm{~s}^{-1}$ showed spiral patterns similar to those in Fig. 3b, indicating inhomogeneous flow even after $10 \mathrm{~min}$ shearing. The floc size seemed to be independent of the concentration of the suspension at those concentrations that were image analyzed. The dilute concentrations ( 0.1 and $0.25 \%)$, however, exhibited totally different structure after shearing at $0.5 \mathrm{~s}^{-1}$. This is discussed in more detail later in this paper.

If we compare the floc sizes from the $10 \mathrm{~min}$ constant shear (Fig. 7) to those measured during the 


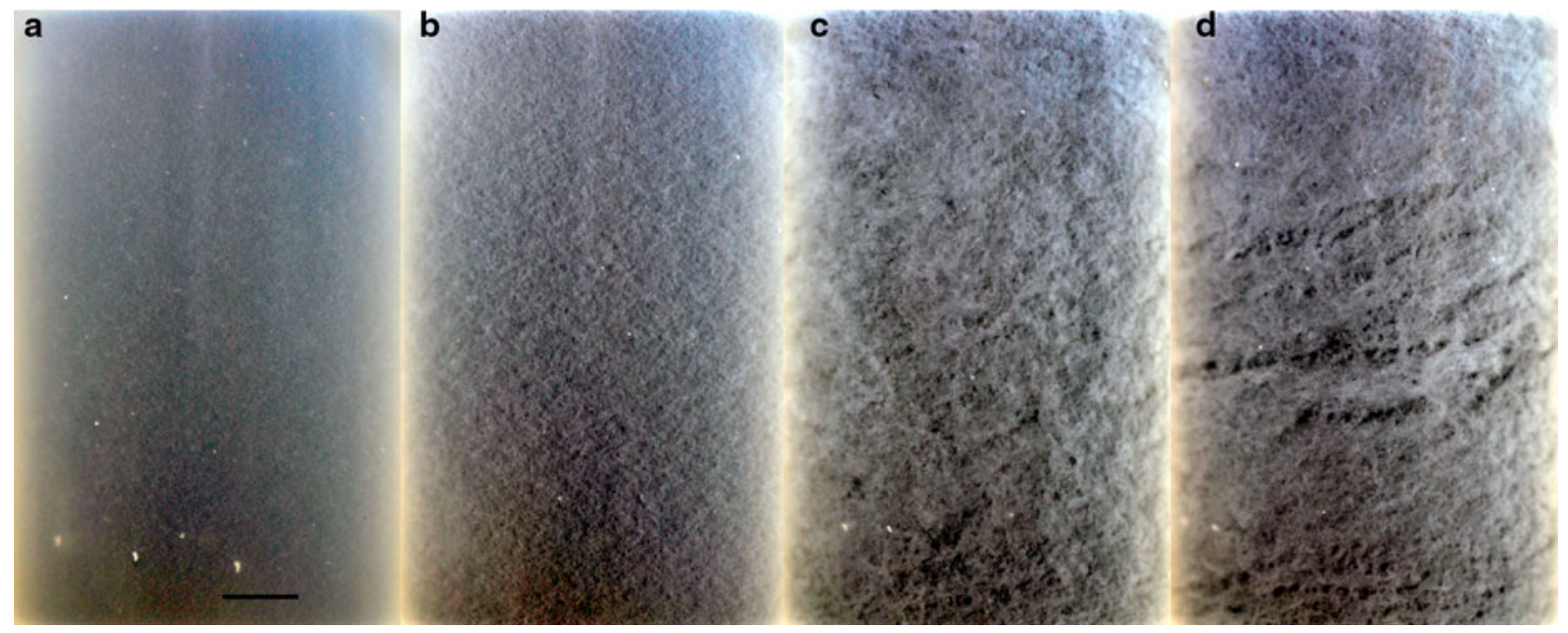

Fig. 6 A qualitative view of the MFC $1 \%$ suspension structure immediately after peak holds at a $500 \mathrm{~s}^{-1}$, b $30 \mathrm{~s}^{-1}$, c $5 \mathrm{~s}^{-1}$, and d $0.5 \mathrm{~s}^{-1}$. The inner cylinder rotates to the left. The length of the scale bar is $5 \mathrm{~mm}$
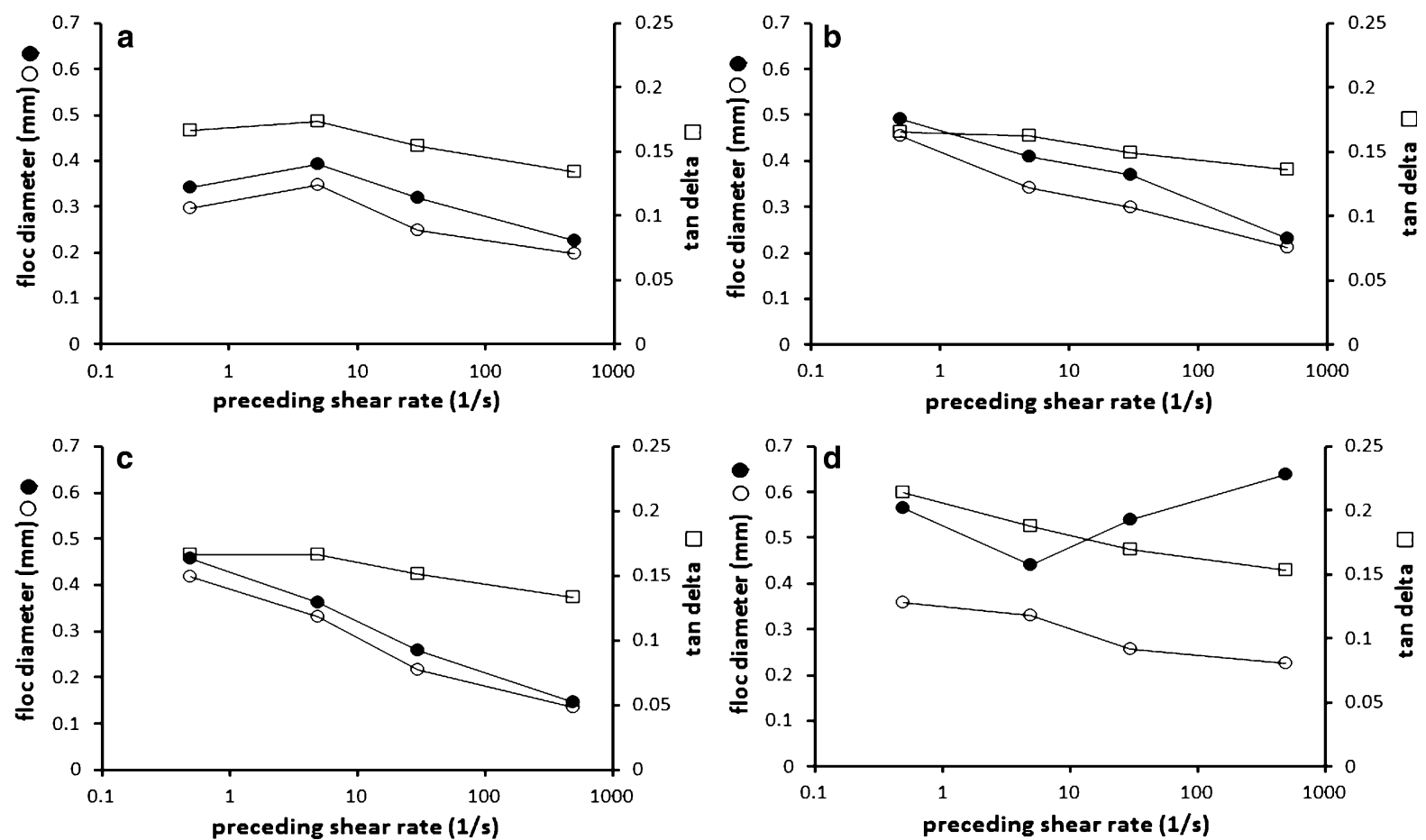

Fig. 7 Floc size in $\mathrm{x}$ (empty circles) and y (filled circles) direction and tan deltas (empty squares) measured $500 \mathrm{~s}$ after peak holds at different shear rates for MFC at a concentration of a $2 \%$, b $1.5 \%$, c $1 \%$, and $\mathbf{d} 0.5 \%$

flow curve (Fig. 4), we see that the high shear rate results from the constant shear measurements fit well to the trend in the flow curve results. At high shear rates, the point time (15 s) of the flow curve was sufficient for the flocs to adapt to the flow conditions and no further change was seen after longer shearing.
Instead, the lower shear rate results measured during the flow curve and after constant shear differed from each other. Some suspensions underwent network breaking in the flow curve at shear rate $5 \mathrm{~s}^{-1}$, and in these cases the floc size was much higher in the flow curve than after constant shear. The biggest difference 

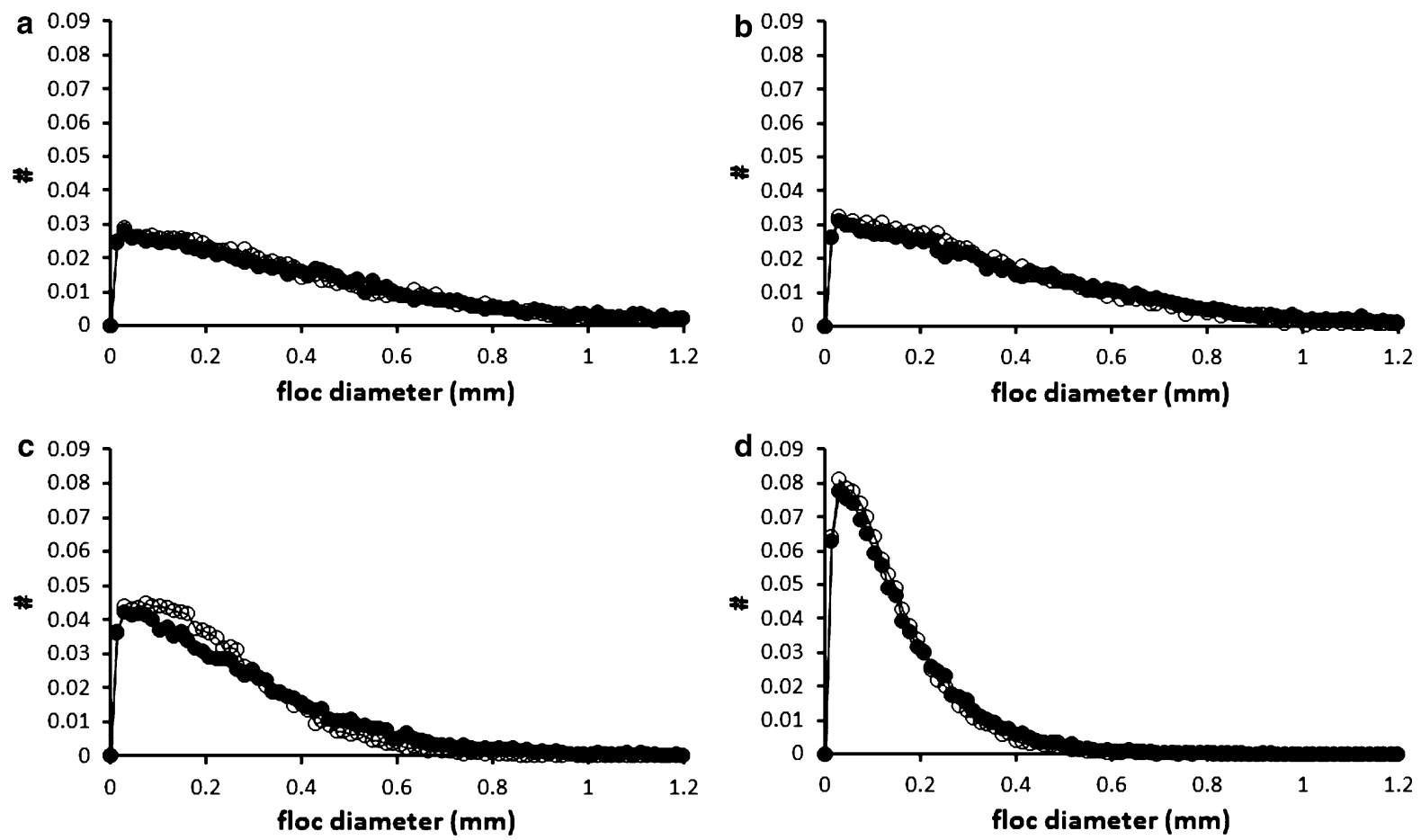

Fig. 8 Floc size distribution in $\mathrm{x}$ (empty circles) and y (filled circles) directions for MFC $1 \%$ after 10 min constant shear at $\mathbf{a} 0.5 \mathrm{~s}^{-1}$, b $5 \mathrm{~s}^{-1}$, c $30 \mathrm{~s}^{-1}$, and d $500 \mathrm{~s}^{-1}$

was at shear rate $0.5 \mathrm{~s}^{-1}$. The lowest shear rate $0.5 \mathrm{~s}^{-1}$ was so low that the point time in the flow curve (15 s) was far too short for the flocs to accommodate to the flow conditions and, therefore, the floc size in the flow curve corresponds to the previous shearing situation.

The tan deltas in Fig. 7 were dependent on the preceding shear interval. Especially, the tan deltas after the two lowest shear rates were higher than the tan deltas after shearing at 30 or $500 \mathrm{~s}^{-1}$. Tan delta is the ratio of loss modulus to storage modulus, and an increasing tan delta indicates increased viscous energy dissipation related to elastic energy storage. At lower shear rates, the floc structure was heterogeneous and contained voids in the MFC network filled with water. The apparently fiber free areas gave rise to the viscous component in relation to the elastic component and increased the tan deltas measured in oscillation time sweeps after constant shear.

The two lowest concentrations $(0.25$ and $0.1 \%)$ failed in the image analysis due to the poor contrast between the flocs and the inner cylinder. Qualitatively, the floc structures from the peak holds at 500, 30, and $5 \mathrm{~s}^{-1}$ were congruent with the higher concentrations, but the structures after constant shear at $0.5 \mathrm{~s}^{-1}$ were of interest. The structures are shown in Fig. 9. At low shear rate $\left(0.5 \mathrm{~s}^{-1}\right)$ and low concentrations $(0.1$ and $0.25 \%)$, MFC formed floc cylinders perpendicular to the direction of flow. The shear rate $0.5 \mathrm{~s}^{-1}$ increased the floc formation by increasing the collisions between the fibers but it was too low to re-disperse the structures. However, the flocculation was not permanent aggregation, since when the shear rate was afterwards raised stepped-wise, the floc chains were gradually re-dispersed, starting from a shear rate of around $5 \mathrm{~s}^{-1}$. Similar floc cylinders started to form during the flow curve for the two lowest concentrations (Fig. 1), but the time scale was so short (point time $15 \mathrm{~s}$ ) that only a few cylinders appeared in the gap.

This kind of shear-induced formation of vorticity aligned floc cylinders in a confined space has been observed, for example, in carbon nanotube suspensions (Lin-Gibson et al. 2004; Hobbie and Fry 2006; Ma et al. 2007) and carbon black gels (Grenard et al. 2011) with optical microscopy. It has been suggested that the cylindrical flocs undergo a log-rolling movement, and they are a consequence of elastic instability and large negative first normal stress differences 

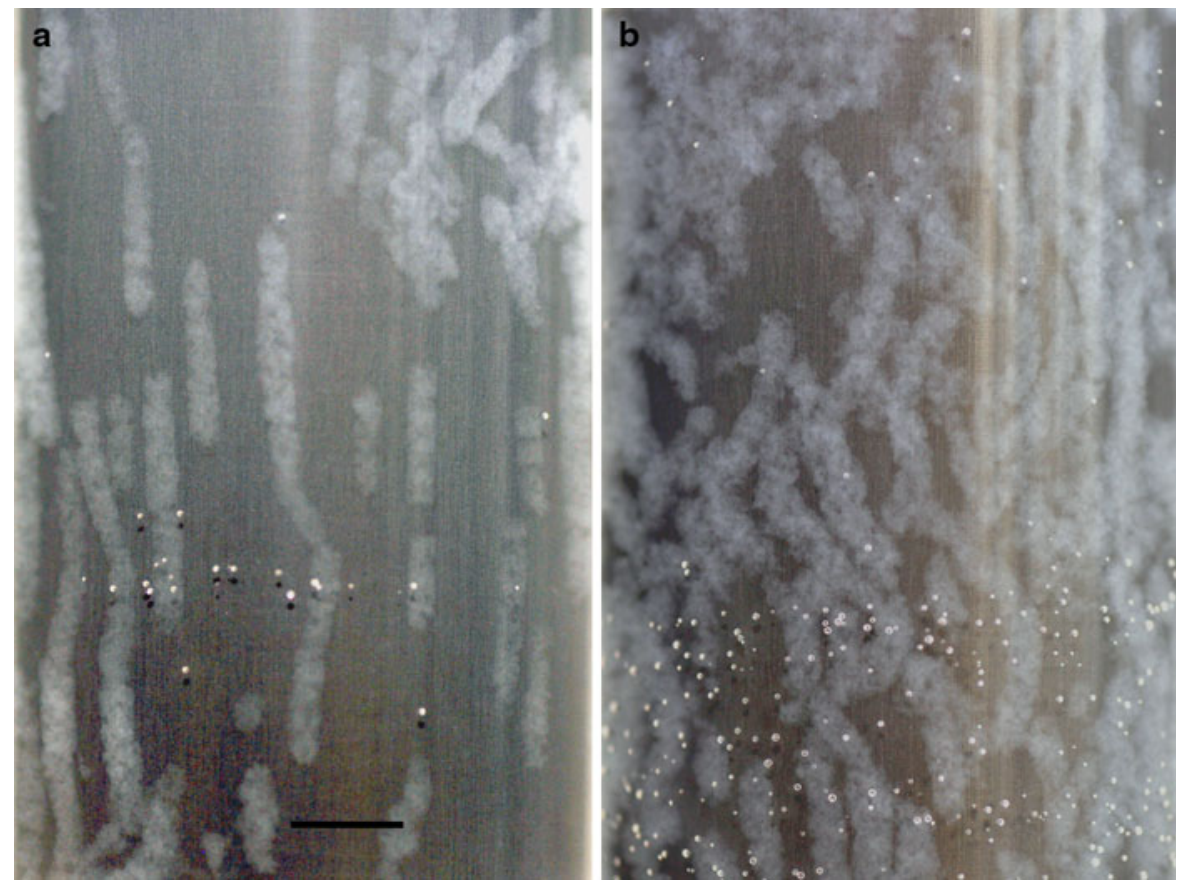

Fig. 9 Photos of MFC at concentration of $0.1 \%$ (on the left hand side) and $0.25 \%$ (on the right hand side) after shearing 10 min at $0.5 \mathrm{~s}^{-1}$. The inner cylinder rotates to the left. The length of the scale bar is $5 \mathrm{~mm}$

(Lin-Gibson et al. 2004; Montesi et al. 2004) or aggregation confined by the gap size (Ma et al. 2007). We assume that the flocs in the MFC suspensions started to grow in the gap by collisions when the shear rate was insufficient to break the interactions between the fibers in the floc, and the links between the fibers in the growing flocs were mostly mechanical. Simultaneously, the flocs became denser. When the floc size reached the gap size, the floc continued to grow perpendicular to the flow, forming a cylinder. From Fig. 9, it can be estimated, however, that the diameter of some of the floc chains was greater than the gap $(1 \mathrm{~mm})$, which is different from the carbon nanotube (Lin-Gibson et al. 2004; Hobbie and Fry 2006; Ma et al. 2007) and carbon black suspensions (Grenard et al. 2011).

\section{Conclusions}

We have studied the suspension rheology and structure of MFC under shear using a combination of a rotational dynamic rheometer and digital imaging. The flow curves of MFC suspensions contained two shear thinning regions and a viscosity plateau between them. A correlation between a certain region in the flow curve and a certain microstructure was found. In the first shear thinning region, the floc size was very small and the floc size distribution was narrow, originating from the previous shearing interval, and no changes in the floc size were detected with imaging system used. Fibers were mechanically entangled, and the fiber network was able to recover from the shear, preserving the overall floc network structure. This floc network structure was related to constant shear stress and decreasing viscosity as a function of shear rate.

In the transition region of the flow curve, the floc size increased rapidly and the size distribution broadened clearly. Visual inspection revealed that fiber free voids appeared in the uniform network and, as a result of this, a small plateau in the flow curve was observed. At this point, the shear was sufficiently high to break the points of contact between the flocculated fibers in the network. At the same time, flow increased the collision frequency between the fibers and thus induced new, denser entanglements within the fiber rich areas. These entanglements were mostly reversible in nature, since the flocs started to break loose 
with an increasing shear rate. This indicates that the surface charge of MFC, although it was quite low, caused repulsion between the fibers and prevented permanent aggregation at this low electrolyte concentration. After the transition, the separated, individual flocs decreased in size with the increasing shear rate and the flocs were rolling and moving relative to each other. This structure was related to shear thinning behavior and increasing shear stress with increasing shear rate. All in all, the structural changes were in good agreement with the shape of the flow curve measured here and previously in other studies.

The suspension structures were also studied at 10 min constant shear at the shear rates $0.5,5,30$ and $500 \mathrm{~s}^{-1}$. After constant shear, the floc size was dependent on the preceding shear interval, and a lower shear rate induced larger flocs. Lower shear rates were related to broader size distribution, indicating heterogeneous macroscopic structure. Larger flocs and a heterogeneous structure caused higher tan delta values, measured in an oscillatory time sweep immediately after the constant shear. A low shear rate increased the viscous part in relation to elastic part of the gel, since voids filled with water appeared in the suspension after slow shearing. At the lowest concentrations, shear induced floc cylinders were formed after shearing at $0.5 \mathrm{~s}^{-1}$.

The structure of the suspension explained the rheological results well, but some restrictions were encountered in the image analysis. First, the two lowest concentrations 0.1 and $0.25 \%$ failed in the image analysis, probably because of the poor contrast between the flocs and inner cylinder in the rheometer. Second, some of the structural changes occurred below the observable size scale. For example, after 10 min constant shear, the structure seemed to recover, since tan delta values were decreasing, although any detectable changes were not seen in the structure. Third, using current imaging methods, high shear rates could not be analyzed without first stopping the shear. To better understand these phenomena, higher resolution and high speed analysis methods would be required.

Acknowledgments This work was a part of the Nanosellu III-project established by the Finnish Centre for Nanocellulosic Technologies. The project is funded by the Finnish Funding Agency for Technology and Innovation (Tekes). Prof. Monika Osterberg, Aalto University, is thanked for the discussions on MFC surface charge properties in aqueous environment.

\section{References}

Agoda-Tandjawa G, Durand S, Berot S, Blassel C, Gaillard C, Garnier C, Doublier J-L (2010) Rheological characterization of microfibrillated cellulose suspensions after freezing. Carbohydr Polym 80:677-686

Barnes HA (1997) Thixotropy-a review. J Non-Newton Fluid Mech 70:1-33

Beghello L (1998) Some factors that influence fiber flocculation. Nord Pulp Pap Res J 13:274-279

Björkman U (2000) Flow of flocculated fibers, 2nd edn. TS Högskoletryckeriet, Stockholm

Björkman U (2003a) Break-up of suspended fibre networks. Nord Pulp Pap Res J 18:32-37

Björkman U (2003b) Stress generation and transmission in suspended fibre networks. Nord Pulp Pap Res J 18:38-43

Björkman U (2005) Floc dynamics in flowing fibre suspensions. Nord Pulp Pap Res J 20:247-252

Björkman U (2006) The metarheology of crowded fibre suspensions. Ann Trans Nord Rheol Soc 14:69-78

Chen B, Tatsumi D, Matsumoto T (2002) Floc structure and flow properties of pulp fiber suspensions. J Soc Rheol Jpn 30:19-25

Derjaguin B, Landau L (1941) Theory of the stability of strongly charged lyophobic sols and of the adhesion of strongly charged particles in solutions of electrolytes. Acta Physicochim URSS 14:633-662

Eronen P, Junka K, Laine J, Österberg M (2011) Interaction between water-soluble polysaccharides and native nanofibrillar cellulose thin films. BioResources 6:4200-4217

Grenard V, Taberlet N, Manneville S (2011) Shear-induced structuration of confined carbon black gels: steady-state features of vorticity-aligned flocs. Soft Matter 7:3920 3928

Herrick FW, Casebier RL, Hamilton JK, Sandberg KR (1983) Microfibrillated cellulose: morphology and accessibility. J Appl Polym Sci: Appl Polym Symp 37:797-813

Hobbie EK, Fry DJ (2006) Nonequilibrium phase diagram of sticky nanotube suspensions. Phys Rev Lett 97:03610110361014

Hubbe MA (2007) Flocculation and redispersion of cellulosic fiber suspension: a review of effects of hydrodynamic shear and polyelectrolytes. BioResources 2:296-331

Hubbe MA, Rojas OJ (2008) Colloidal stability and aggregation of lignocellulosic materials in aqueous suspension: a review. BioResources 3:1419-1491

Iotti M, Gregersen OW, Moe S, Lenes M (2011) Rheological studies of microfibrillar cellulose water dispersions. J Polym Environ 19:137-145

Karema H, Salmela J, Tukiainen M, Lepomäki H (2001) Prediction of paper formation by fluidisation and reflocculation experiments. FRC Symposium on the Science of Papermaking, 17-21 Sept, p 29

Karppinen A, Vesterinen A, Saarinen T, Pietikäinen P, Seppälä J (2011) Effect of cationic polymethacrylates on the rheology and flocculation of microfibrillated cellulose. Cellulose 18:1381-1390

Kerekes RJ (2006) Rheology of fibre suspensions in papermaking: an overview of recent research. Nord Pulp Pap Res J 21:598-612 
Kerekes RJ, Soszynski RM, Tam Doo PA (1985) The flocculation of pulp fibres. In: Punton V (ed) Transactions of the 8th fundamental research symposium. Mechanical Engineering Publications, Sept 1985, p 265

Lin-Gibson S, Pathak JA, Grulke EA, Wang H, Hobbie EK (2004) Elastic flow instability in nanotube suspensions. Phys Rev Lett 92:048302-1-048302-4

Lowys M, Desbrières J, Rinaudo M (2001) Rheological characterization of cellulosic microfibril suspensions. Role of polymeric additives. Food Hydrocoll 15:25-32

Ma A, Mackley M, Rahatekar S (2007) Experimental observation on the flow-induced assembly of carbon nanotube suspensions to form helical bands. Rheol Acta 46:979-987

Mewis J, Wagner NJ (2009) Thixotropy. Adv Colloid Interface Sci 147-148:214-227

Montesi A, Pena AA, Pasquali M (2004) Vorticity alignment and negative normal stresses in sheared attractive emulsions. Phys Rev Lett 92:058303-1-058303-4

Ono H, Shimaya Y, Sato K, Hongo T (2004) 1H spin-spin relaxation time of water and rheological properties of cellulose nanofiber dispersion, transparent cellulose hydrogel (TCG). Polym J 36:684-694

Pääkkö M, Ankerfors M, Kosonen H, Nykänen A, Ahola S, Österberg M, Ruokolainen J, Laine J, Larsson PT, Ikkala O, Lindström T (2007) Enzymatic hydrolysis combined with mechanical shearing and high-pressure homogenization for nanoscale cellulose fibrils and strong gels. Biomacromolecules 8:1934-1941

Saarikoski E, Saarinen T, Salmela J, Seppälä J (2012) Flocculated flow of microfibrillated cellulose water suspensions: an imaging approach for characterisation of rheological behaviour. Cellulose. doi:10.1007/s10570-012-9661-0

Saito T, Nishiyama Y, Putaux J, Vignon M, Isogai A (2006) Homogeneous suspensions of individualized microfibrils from TEMPO-catalyzed oxidation of native cellulose. Biomacromolecules 7:1687-1691

Solomon MJ, Spicer PT (2010) Microstructural regimes of colloidal rod suspensions, gels, and glasses. Soft Matter 6: $1391-1400$

Swerin A, Ödberg L, Lindström T (1990) Deswelling of hardwood kraft pulp fibers by cationic polymers. Nord Pulp Pap Res J 5:188-196

Turbak AF, Snyder FW, Sandberg KR (1983) Microfibrillated cellulose, a new cellulose product: properties, uses, and commercial potential. J Appl Polym Sci Appl Polym Symp 37:815-827

Vartiainen J, Pöhler T, Sirola K, Pylkkänen L, Alenius H, Hokkinen J, Tapper U, Lahtinen P, Kapanen A, Putkisto K, Hiekkataipale P, Eronen P, Ruokolainen J, Laukkanen A (2011) Health and environmental safety aspects of friction grinding and spray drying of microfibrillated cellulose. Cellulose 18:775-786

Vermant J, Solomon MJ (2005) Flow-induced structure in colloidal suspensions. J Phys Condens Matter 17:R187-R216

Verwey EJW, Overbeek JTG (1948) Theory of the stability of lyophobic colloids: the interaction of particles having an electric double layer. Elsevier, New York

Wågberg L, Nordqvist T (1999) Detection of polymer induced flocculation of cellulosic fibres by image analysis. Nord Pulp Pap Res J 14:247-255 